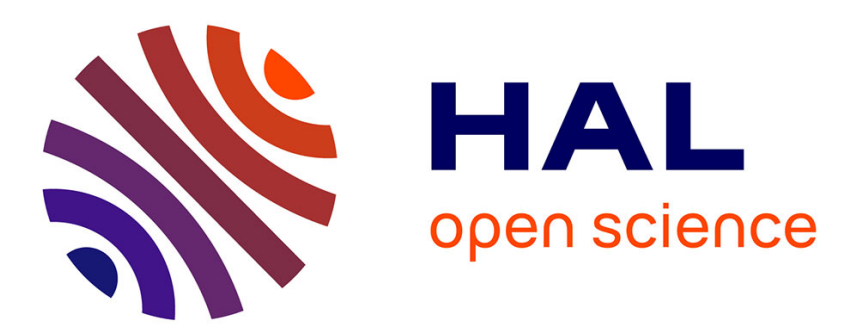

\title{
Solid state intracavity laser absorption spectroscopy spectro temporal transient behavior of a Ti:Al2O3 laser
}

\author{
A. Kachanov, A. Charvat, F. Stoeckel
}

\section{To cite this version:}

A. Kachanov, A. Charvat, F. Stoeckel. Solid state intracavity laser absorption spectroscopy spectro temporal transient behavior of a Ti:Al2O3 laser. Journal de Physique IV Proceedings, 1994, 04 (C4), pp.C4-619-C4-624. 10.1051/jp4:19944166 . jpa-00252623

\section{HAL Id: jpa-00252623 https://hal.science/jpa-00252623}

Submitted on 1 Jan 1994

HAL is a multi-disciplinary open access archive for the deposit and dissemination of scientific research documents, whether they are published or not. The documents may come from teaching and research institutions in France or abroad, or from public or private research centers.
L'archive ouverte pluridisciplinaire HAL, est destinée au dépôt et à la diffusion de documents scientifiques de niveau recherche, publiés ou non, émanant des établissements d'enseignement et de recherche français ou étrangers, des laboratoires publics ou privés. 


\title{
Solid state intracavity laser absorption spectroscopy spectro temporal transient behavior of a $\mathrm{Ti}^{-\mathrm{Al}_{2}} \mathrm{O}_{3}$ laser
}

\author{
A. KACHANOV ${ }^{(1)}$, A. CHARVAT and F. STOECKEL \\ Laboratoire de Spectrométrie Physique, Université Joseph Fourier de Grenoble, URA 08 du CNRS, BP. 87, \\ 38402 Saint-Martin-d'Hères cedex, France
}

Intracavity laser absorption spectroscopy (ICLAS) is known as one of the most sensitive techniques for very weak absorption measurements [1]. This technique is based on the time dependence and the high sensitivity of a highly multimode laser to intracavity frequency dependent losses when the linewidth of the absorbing species is larger than the mode spacing and narrower than the gain curve. Until now, the gain medium of most of the lasers (dye lasers, color center lasers, ...) used in the ICLAS technique had shorter relaxation times than the photon lifetimes in the cavity and the dynamics of such lasers is now well understood at least when the coupling between modes can be neglected.

The recent development of solid state lasers opens a new field for ICLAS mainly for applications in the near infrared (spectroscopy, trace analysis of gases, optical diagnostic in plasma, ...). In turn the use of this new class of lasers needs a better understanding of their spectro-temporal behaviour and of the limitation of the sensitivity in the ICLAS technique. In this work we analyse both experimentally and by computer modelling, the transient behaviour and the sensitivity limitations of the solid state laser on the example of a Ti:sapphire laser.

Main features of the new media such as Ti:Sapphire, Chromium doped forsterite, Cr:LICAF, Cr:LISAF, $\mathrm{Cr}^{4+}:$ YAG etc. are: a very broad homogeneous linewidth, low threshold pumping powers, a high optical quality and high stability.

A spectral range from 0.7 to $1.6 \mu$ can be covered by a few crystals. which is very attractive for the infrared high resolution spectroscopy, trace analysis of gases and optical diagnostics in the industry. The possibility of a diode pumping may give in the nearest future a new generation of the intracavity laser spectrometers promising to be very compact, low cost, low power consuming and very simple in use, like conventional classical spectrometers.

(1) on leave from the Institute for Spectroscopy, Russian Academy of Sciences, 142092 Troitsk, Moscos Region, Russia 
For this reason it seems to be very important to evaluate the possible performance of the solid-state intracavity spectrometers, and to understand if there are new problems and what the limitations in using them are for the ICLAS.

In this work we investigated the time dependence of the intracavity absorption for a Ti:Sapphire laser, which is a good representative of all the varieties of the solid state materials both in the short and in the long generation time domains. The influence of the nonlinear effects limiting the maximum sensitivity of the solid-state based ICLAS was also studied.

It is known (see review paper [1]), that if the spectrum of a laser with a homogeneously broadened gain profile is displayed in a logarithmic scale, then the intracavity absorption is represented by $N \sigma(v) \cdot c \cdot t_{\mathrm{g}}=N \sigma(v) L_{e q}$, where $N$ is the density of absorbing species, $\sigma(v)$ is the absorption cross section, $c$ is the velocity of light, and $t_{g}$ is the generation time (the time from the beginning of the laser generation to the moment of observation). The product $c \cdot t_{g}$ may be defined as an equivalent absorption path $L_{e q}$. The linear growth of the equivalent absorption path is limited by the spontaneous emission at a characteristic time $\tau_{s p} \cong\langle M\rangle / \gamma$, where $\langle M\rangle$ is the average number of photons in the mode and $\gamma$ is the intracavity loss rate. The characteristic time $\tau_{s p}$ being expressed through the parameters of the cavity and of the active medium, gives an estimate for the fundamental limit of the sensitivity for a given intracavity spectrometer:

$$
\tau_{s p} \cong \frac{(\eta-1)^{2} w_{0}^{4} L}{32 \sigma_{0}^{2} \Gamma^{2} \tau^{2} c^{3} \ln \left[R^{-1}\left(1-T_{i}\right)^{-2}\right]},
$$

where $L$ is the cavity length, $w_{0}$ is the laser beam radius inside the active medium, $\mathrm{R}$ and $T_{i}$ represent the output mirror reflectance and the intracavity losses, $\eta$ is a pump power excess above the threshold, and $\sigma_{0}, \Gamma$ and $\tau$ are the emission cross section, the gain linewidth and the fluorescense lifetime of the active medium [2]. For a typical Ti:Sapphire laser it gives $\tau_{s p} \cong 0.7 \mathrm{~s}$, or $L_{e q} \cong 210,000 \mathrm{~km}$ !

The solid state lasers have relatively long cavity build up times and often strong relaxation oscillations. In order to apply them for the ICLAS we should know the answer to three main questions:

i) what is the generation time for the solid state laser?

ii) is there any deviation of the linear growth of the absorption, due to the relaxation ascillations?

iii) is it posible to reach the fundamental limit of the sensitivity defined by the spontaneous emission?

The schematics of our Ti:Sapphire intracavity spectrometer is shown in Fig. 1.

This setup makes possible to record the intracavity absorption spectra with a variable time delay $t_{g}$ from the leading edge of the pumping radiation and thus to study the time dynamics of the intracavity absorption [1]. More detailed description of the setup may be found in [3]. 


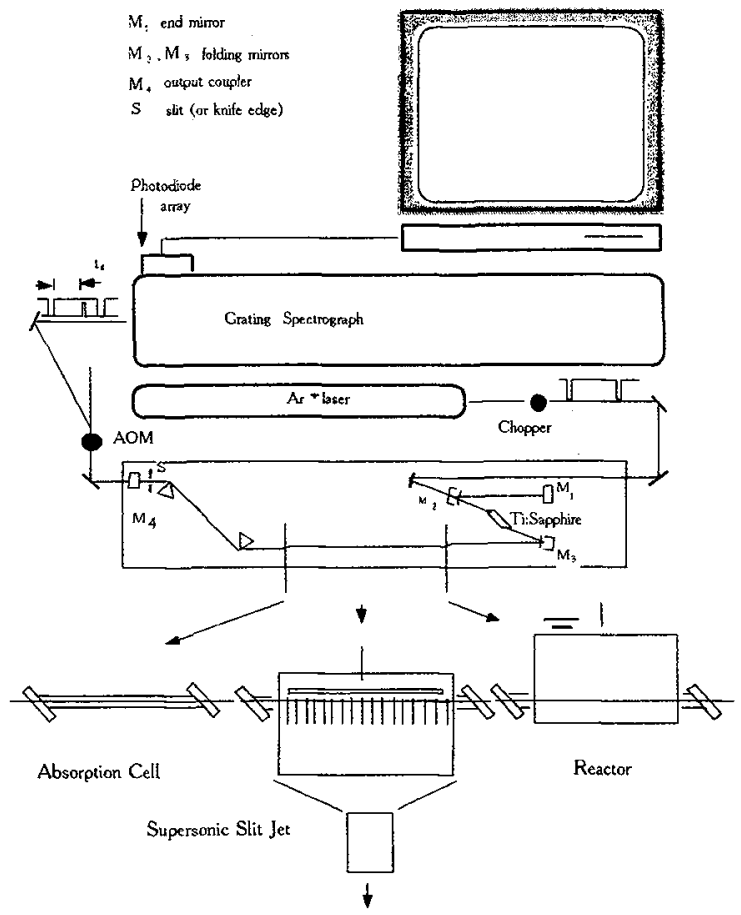

Fig. 1. Ti:Sapphire intracavity absorption spectrometer.

Both an experimental study and a computer modelling of the intracavity absorption in the region of a generation build-up and the relaxation oscillations have shown that the relaxation oscillation have no effect on the linear dependence of the intracavity absorption on the generation time. The absorption can be expressed by a modified Lambert-Beer law with an equivalent path length given by $c\left(t_{t h}-t_{g}\right)$.in which $t_{t h}$ is an offset time given by the time needed by the inversion to reach the threshold.

For long genearation times the sensitivity of the ICLAS in most of the lasers used before (mostly dye lasers and color center lasers) was limited by nonlinear mode interactions. As a result, the absorption stops growing at times $\tau_{m}$ which is two or three orders of magnitude lower than $\tau_{s p}$ due to the spontaneous emission. Solid state media have much longer fluorescence lifetimes and thus much higher damping of the oscillations of the nonlinear polarization on the intermode frequencies. We can expect therefore, that the nonlinear intermode interaction in the solid-state lasers should be two or three orders of magnitude weaker than in the dye and color center lasers [2]. It means that we can expect to reach the sensitivity approaching the fundamental limit for these lasers.

Nevertheless even for a generation time approaching $1 \mathrm{~ms}$, some deviations from the linear time dependence of the equivalent absorption path were observed in our linear cavity. When the total output power was recorded with a high frequency photodiode, a modulation on the intermode frequency was observed with a modulation depth as high as $40 \%$. This modulation may be due to the Kerr effect in the 
Ti:Sapphire crystal, which may work as a passive mode-locker [4]. This partial mode locking means a coupling between the modes and may explain qualitatively the observed nonlinearity in the intracavity absorption. More detailed researches are needed in order to understand better this nonlinear dynamics of the solid state lasers.

After we had modified the linear configuration of our Ti:Sapphire laser into a ring configuration similar to [5] in order to receive unidirectional travelling wave operation, the linear growth of the intracavity absorption was observed for much longer times. In Fig. 2 the time dependence of the absorption for two atmospheric water vapor lines is shown in a time range from 0 to $3 \mathrm{~ms}$. No deviations from the expected linear dependence may be noticed for a generation time $3 \mathrm{~ms}$ which corresponds to an equivalent absorption path of $900 \mathrm{~km}$.

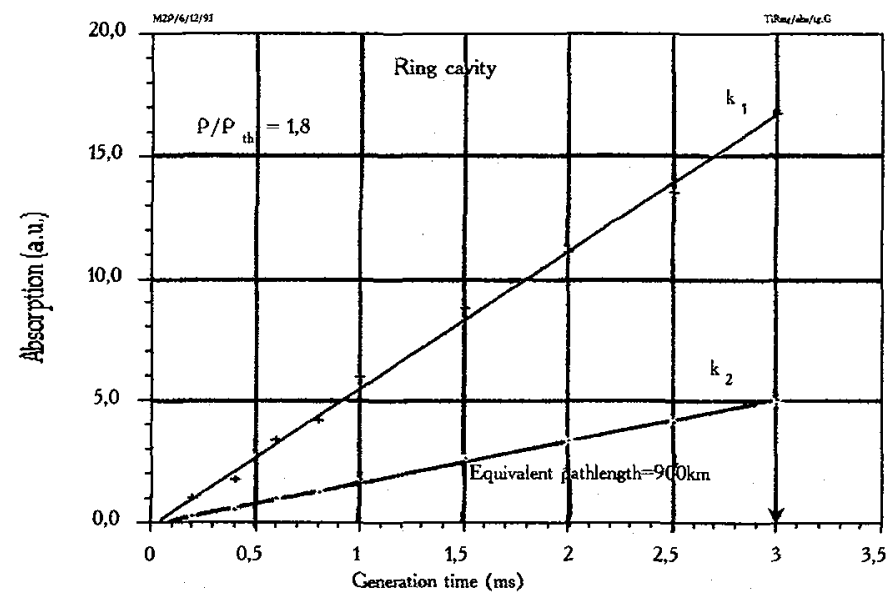

Fig. 2. Absorption versus generation time $\mathrm{t}_{\mathrm{g}}$ with a Ti:Sapphire ring intracavity spectrometer for two athmospheric water vapor lines.

Unfortunately, we were not able to record the absorption spectrum in a ring laser for longer generation times due to the birefringence of the Ti:Sapphire crystal. This birefringence gives a frequency selective. loss similar to that given by a Lyot type birefringent filter and makes the spectrum at higher generation times too narrow. We can nevertheless estimate the limits of the linear growth of the sensitivity by measuring, rather than the absorptin of a line, the dependence of the width of the generation spectrum, which should be inversely proportional to the square root of the generation time[1]. Such a measurement for our Ti:Sapphire ring laser shows, that its sensitivity increases linearly with generation time at least until $40 \mathrm{~ms}$ which is equivalent to an absorption path of $12,000 \mathrm{~km} .$.

Parasitic interference fringes are a serious problem in a standing wave solid state ICLAS spectrometers. They prevent the observation of weak absorption lines for generation times approaching $1 \mathrm{~ms}$. It is nevertheless possible to obtain spectra of a very high quality with our standing wave laser for generation times as high as $100-200 \mu \mathrm{s}$. As an example, we show in Fig. 3 the absorption spectra of the overtone 
transitions of the $\mathrm{CH}_{4}$ molecule at room temperature (upper spectrum) and cooled in the supersonic free slit jet (lower spectrum). The equivalent path length in both spectra was $30 \mathrm{~km}$.
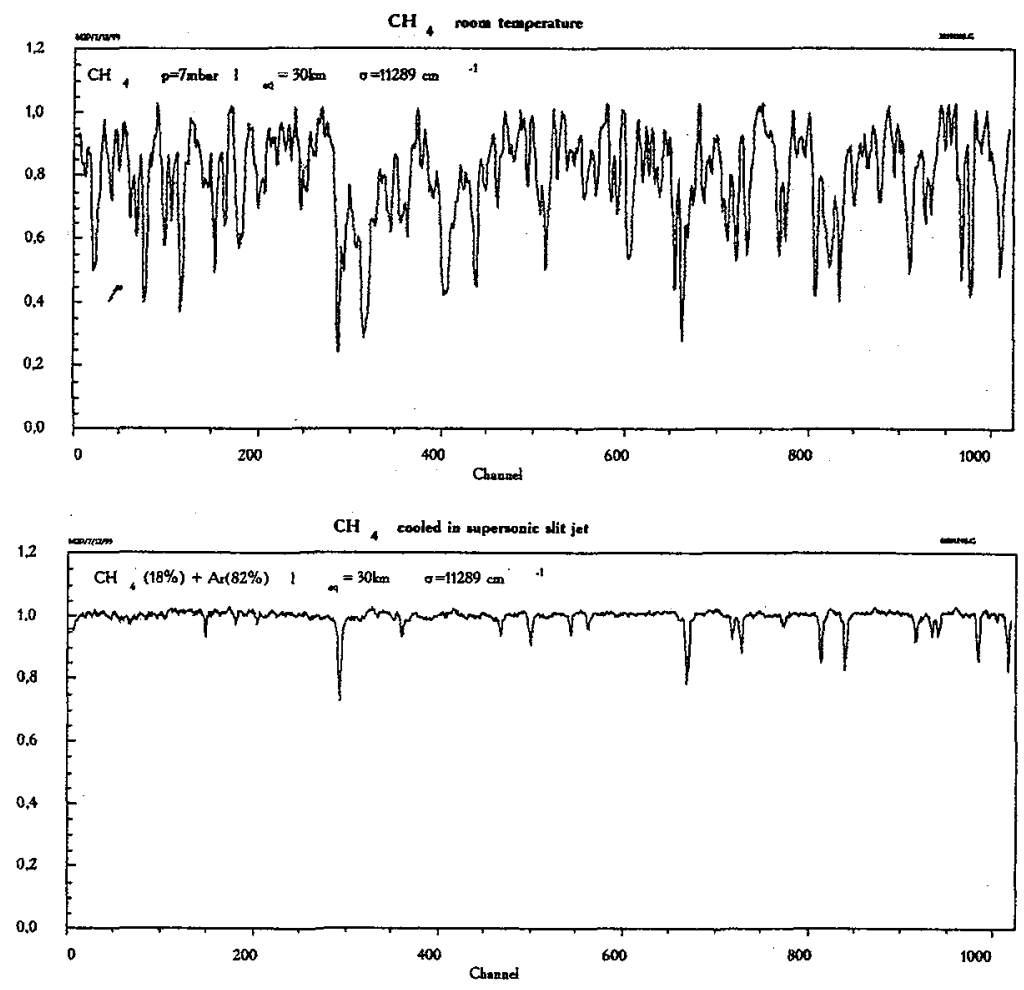

Fig. 3. Intracavity absorption spectra of the $\mathrm{CH}_{4}$ molecule at room temperature (upper trace) and in the supersonic jet.(lower trace) near $885.58 \mathrm{~nm}$

In the ring configuration no interference fringes were observed in the spectrum either at the generation time of $3 \mathrm{~ms}$, or even for a time of $40 \mathrm{~ms}$.

We can conclude now that the intracavity detection free of the interference fringes was demonstrated at least for a generation time of $3 \mathrm{~ms}$. We can expect then, that quantum noise limited detection threshold for weak absorptions can be achieved with a solid state laser ICLAS spectrometer similarly to what was demonstrated for a dye laser ring ICLAS spectrometer [5]. It means, that if we assume the same conditions as in [2] for the registration system and a signal averaging time of 1s, then an absorption line with a depth of about $0.1 \%$ can be detected with an equivalent absorption path length of $1000 \mathrm{~km}$. It means that the minimum detectable absorption coefficient may be $\cong 5 \cdot 10^{-11} \mathrm{~cm}^{-1}$. Using for the detection several absorption lines which are inside the spectral window of the intracavity spectrometer rather than a 
single line and aplying a data processing algorithm described in [2], we can even expect to decrease this quantity several times. The estimate for the detection limitfor the absorption is then of $\cong 1 \cdot 10^{-11} \mathrm{~cm}^{-1}$.

Low power diode pumping for several solid-state media has already been demonstrated like for example for a Cr:LICAF laser working in the same range than a Ti:Sapphire laser. The diode pumping seems to be also possible for a $\mathrm{Cr}^{4+}:$ YAG laser which is tunable in the range 1.35-1.60 $\mu$. Such a possibility makes the solid-state ICLAS a potential competitor with a diode laser based spectrometer for atmospheric pollution monitoring, or gas purity control in the industry for example. A diode-pumped solid-state intracavity spectrometer promises to be easier in operation, of a lower price, and more easily tunable over a very broad spectral range without changing the active medium, than a high sensitivity diode laser spectrometer using a high frequency modulation detection technique with a multipass cell.

Let us nowestimate a detection threshold for a diode laser spectrometer. From the litterature we know, the best value for the absorption demonstrated is of the order of $10^{-6}$. Assuming an optical path length in a multipass cell of $25-30$ meters, as it is commonly used, we obtain a detection threshold of $\cong 3 \cdot 10^{-10} \mathrm{~cm}^{-}$ ${ }^{1}$, that is one order of magnitude less than our estimate for the intracavity spectrometer. In the other hand ICLAS spectrometers may have a potential to win one or more orders of magnitude by increasing the generation time until $30-300 \mathrm{~ms}$, which gives a detection limite for the absorption of $10^{-12}-10^{-13} \mathrm{~cm}^{-1}$.

As a conclusion, we want to point out, that a solid-state Ti:Sapphire intracavity spectrometer with an equivalent absorption path length of $900 \mathrm{~km}$ was demonstrated. A comparison with diode laser spectrometers for highly sensitive gas analysis was made. One to two orders of magnitude lower detection limits, better versatility and simplicity can be expected for future all solid state ICLAS spectrometers working in the near infrared.

\section{REFERENCES}

[1] A. CAMPARGUE, F. STOECKEL and M. CHENEVIER

High sensitivity intracavity laser spectroscopy : applications to the study of overtone transitions in the visible range. Spectrochimica Acta Rev. Vol. 13, pp 69-88, 1990.

[2] A. KACHANOV, A. CHARVAT, F. STOECKEL Intracavity laser spectroscopy with vibronic solid state lasers :

I : Spectro temporal transient behaviour of a Ti:Sapphire laser.

II : Influence of the non linear mode coupling on the maximum sensitivity of a Ti:Sapphire laser. To be published.

[3] A. CHARVAT, A. KACHANOV, A. CAMPARgUE, D. PERMOGOROV, and F. STOECKEL, "High sensitive intracavity absorption spectroscopy of $\mathrm{CHD}_{3}$ in the near infrared with a titanium:sapphire laser," Chem. Phys. Lett. 214, 495-501, (1993)

[4] D.E. SPENCE, P.N. KEAN and W. SIBBET

Opt lett. 16, 324, (1991)

[5] A. KACHANOVA.A, T.V.PLAKHOTNIK, Intracavity spectrometer with a ring trawelling-wave dye laser: Reduction of detection limit, Optics Commun. 46, N4,.257-261, (1983). 